\title{
MUTIPLE STAR FORMATION FROM N-BODY. SYSTEM DECAY
}

\author{
R. S. HARRINGTON \\ U.S. Naval Observatory, Washington, D.C.
}

\begin{abstract}
RESUMEN
Se ha seguido numériçamente el decaimientó dinámico de sistemàs de $\mathrm{N}$ cuerpós, casuales, inestables y de energía negativa para investigar la estadística de los productos estables del decaimiento. De sistemas iniciales cuádruples, el $20 \%$ de los productos fueron triples estables, mientras que el $30 \%$ resultó de sistemas inicialmente quíntuples. Estos números son consistentes con la proporción de triples a binarias, especialmente para los sistemas de multiplicidad inicial alta.
\end{abstract}

\begin{abstract}
The dynamical decays of random, unstable, negative-energy, n-body systems have been followed numerically, to investigate the statistics of stable decay products. From initial quadruple systems, about $20 \%$ of the products were stable triples while $30 \%$ resulted from initial quintuple systems. These numbers are consistent with the observed ratio of triples to binaries, especially for the higher-multiplicity initial systems.
\end{abstract}

One possibility for the formation of multiple stars is that they are the end products of the dynamical decay of larger stellar systems. Van Albada (1968) first presented examples of this mechanism, and about $30 \%$ of his cases produced apparently stable triples (the rest producing binaries), in good agreement with observed frequencies. However, a sampling of products from a larger number of systems is desirable for a careful check of the statistics of decay products.

Unfortunately, the computer time required to integrate a large number of many-body systems would be prohibitive at most facilities. In addition, the statistics of stable systems with more than 3 components is not well known. Therefore, on the assumption that large systems do not decay explosively, but rather through a sequence of single escapes, the decay of relatively small systems can be followed, with the objective of examining only the statistics of triples and binaries.

An early effort in this direction (Harrington 1974) considered only the decay of 4-body systems. However, in that study there were several examples of decay to two binaries, indicating that decay can proceed through the escape of binaries. Therefore, a second experiment was carried out with 5-body systems (Harrington 1975).

The procedure in both experiments was to pick a large number of random systems, numerically integrate them until they decayed at least as far as a triple, and count the numbers of resulting binaries and triples. Triples were tested for stability by using the criteria discussed earlier in these Proceedings (Harrington 1977). The initial conditions were picked so that in general the energy was negative, the system was unstable, there was a realistic range of masses, the system had positive angular momentum, and the virial theorem was approximately satisfied. Both 2- and 3-dimensional systems (100 apiece) were considered in the 4-body experiment; just 120 3-dimensional systems were considered in the 5-body experiment.

The results are given in Table 1 . The rate tabulated is defined as the fraction of binaries plus triples that are triples. The 2-dimensional results are unrealistic and are included only for comparison. 
TABLE 1

\begin{tabular}{lccc}
\hline Model & Binaries & Triples & Rate \\
\hline 4-body 2-dimensional & 44 & 9 & $17 \%$ \\
4-body 3-dimensional & 49 & 13 & $21 \%$ \\
5-body 3-dimensional & 56 & 23 & $30 \%$ \\
\hline
\end{tabular}

The lower rate for the 4-body 3-dimensional experiment may reflect the different distributions of initial conditions from those used in the 5-body experiment, but more likely it is indicative of the effect of the fifth body.
The rates, particularly for the 5-body experiment, are in good agreement with the observed results discussed in these Proceedings. Therefore it can be concluded that the dynamical decay of small stellar systems does produce stable triple systems in proportions consistent with observed frequencies.

\section{REFERENCES}

Harrington, R. S. 1974, Celes. Mech., 9, 465.

Harrington, R. S. 1975, A.J., 80, 1081.

Harrington, R. S. 1977, IAU Colloquium No 33 Rev. Mex. Astron. Astrof., 3, 139

van Albada, T. S. 1968, Bull. Astr. Inst. Netherl., 19, 479. 